\title{
99mTc sestamibi SPECT: a possible tool for early detection of breast cancer lesions with high bone metastatic potential
}

\author{
Nicoletta Urbano ${ }^{\ddagger 1}$, Manuel Scimeca ${ }^{\ddagger 2,3}$, Elena Bonanno ${ }^{3,4,5}$ \& Orazio Schillaci*,2,6 \\ ${ }^{1}$ Nuclear Medicine, Policlinico "Tor Vergata", viale Oxford, 81, 00133, Rome, Italy \\ 2Department of Biomedicine \& Prevention, University of Rome "Tor Vergata", Via Montpellier 1, 00133 Rome, Italy \\ ${ }^{3}$ IRCCS San Raffaele, Rome, Italy \\ ${ }^{4}$ Department of Experimental Medicine \& Surgery, University of Rome "Tor Vergata", Via Montpellier, 1, 00133 Rome, Italy \\ ${ }^{5}$ IRCCS Neuromed Lab. "Diagnostica Medica" \& "Villa dei Platani", Avellino, Italy \\ ${ }^{6}$ IRCCS Neuromed, Pozzilli, Italy \\ *Author for correspondence: Tel.: + 390620902419; orazio.schillaci@uniroma2.it \\ ${ }^{\ddagger}$ Authors contributed equally
}

"The early identification of lesions with high metastatic potential by ${ }^{99 \mathrm{~m}} \mathrm{Tc}$ sestamibi high-resolution SPECT analysis could be considered a new frontier for diagnosis and/or therapy of breast lesions."

First draft submitted: 24 September 2018; Accepted for publication: 23 October 2018; Published online: 8 January 2019

Keywords: breast cancer $\bullet$ breast osteoblast-like cells $\bullet$ molecular imaging $\bullet$ SPECT $\bullet{ }^{99 m}$ Tc sestamibi

Breast cancer is one of the most common cancers in women, with more than 1,300,000 cases and 450,000 deaths each year worldwide [1]. These tumors display an extraordinary inclination to grow in bone [1]. Indeed, about $10 \%$ of all breast cancer patients without evidence of bone metastases at the time of diagnosis will have a first relapse in bone within 5 years of their primary diagnosis [2]. Furthermore, approximately $65-75 \%$ of patients with advanced breast cancer will develop bone metastases [2]. Bone involvement in breast cancer can heavily affect patients' quality of life because of pathologic bone fractures, bone pain, hypercalcemia and spinal cord compression. Additionally, these complications contribute significantly to the cost of care [3].

A recent discovery about breast osteotropism demonstrated the presence of cells showing an osteoblast-like phenotype in breast cancer tissues [4,5]. The presence of these cells (breast osteoblast-like cells [BOLCs]) was described by Scimeca et al., for the first time, in 2017 [4]. Specifically, in situ studies demonstrated that during carcinogenesis some breast epithelial cells undergo epithelial-mesenchymal transition (EMT) phenomenon acquiring the molecular and morphological characteristics of mesenchymal cells [6]. In addition, during cancer progression, the local expression of osteoblast differentiation factors, such as BMP-2 [7] and/or PTX3 [8], were responsible for BOLCs generation $[4,9]$. Of note, both ex vivo and in vivo studies demonstrated that BOLCs not only displayed the morphological appearance of osteoblasts, but also they were able to share with them their functional role $[4,9]$. It was found that BOLCs were characterized by the expression of cell markers such as receptor activator of nuclear factor $\mathrm{\kappa}-\mathrm{B}$ ligand (RANKL), RUNX2 and vitamin D receptor, all known as responsible for the production of breast microcalcifications made of hydroxyapatite (HA), a process that similarly occurs during bone mineralization by real osteoblasts [10]. Thus, the presence of HA microcalcifications and/or BOLCs could be considered as new negative prognostic factors of breast cancer. The correlation between the presence of BOLCs in the primary lesions and the development of bone metastasis within 5 years of the cancer diagnosis [4] suggests the hypothesis that BOLCs form at the primary lesion, detach from tumor and colonize the bone, triggering resorption osteoclasts mediated via RANK-RANKL. Here, we report a scientific dissertation about the possible use of $99 \mathrm{mTc}$ sestamibi high-resolution single-photon emission computed tomography (SPECT) to early detection of breast cancer lesions with high propensity to form bone metastasis. 
From the clinical point of view, the presence of BOLCs in breast cancers with high propensity to develop bone metastasis opens new perspectives in the diagnosis and management of breast cancer patients. In particular, chemical-physical and biological characteristics of BOLCs can be exploited for the early identification of the lesions that will develop bone metastasis.

In this context, the ${ }^{99 \mathrm{~m}} \mathrm{Tc}$ sestamibi SPECT analysis could represent an extraordinary possibility to identify both primary and metastatic lesions characterized by the presence of BOLCs. Indeed, distribution and pharmacokinetics of ${ }^{99 \mathrm{~m}} \mathrm{Tc}$ sestamibi appears particularly suitable for in vivo detection of these cells. Chemically, ${ }^{{ }^{9} \mathrm{~m}} \mathrm{Tc}$ sestamibi is a lipophilic cation member of the isonitrile family (hexakis 2-methoxyisobutyl isonitrile) that accumulates in cytoplasm of breast cells as a result of passive diffusion across the plasma membrane [11]. Once in the cytoplasm, ${ }^{99 \mathrm{~m}}$ Tc sestamibi can accumulate inside mitochondria due to their negative membrane potential [11]. Thus, the cationic nature of this molecule makes the accumulation of sestamibi inside mitochondria closely related to those cell metabolic activities that increase the negative plasma membrane potential. Noteworthy, experimental studies showed an increase of the amounts of high transmembrane potential mitochondria in osteoblast cells when treated with molecules able to stimulate the mineralization process [12]. Because of high internal negativity, lipophilic compounds with a delocalized positive charge as sestamibi will be taken up by mitochondria to a greater extent than by other organelles, including the plasma membrane. Based on these evidences, we can speculate an increase of sestamibi uptake in breast lesions with high bone metastatic potential due to the presence of numerous BOLCs actively involved in the production of HA crystals. Thus, the ${ }^{99 \mathrm{~m}} \mathrm{Tc}$ sestamibi high-resolution SPECT analysis, a noninvasive and painless imaging examination that uses small doses of radiation, generally consisting of 4-12 images, allows for rapid interpretation and efficient workflow and might high point, quickly and at low cost, the osteotropic breast lesions. At the state of art, we know that ${ }^{99 \mathrm{~m}} \mathrm{Tc}$ sestamibi distributes homogeneously in the normal breast, regardless of the degree of breast density as assessed by mammograms, and it is usually of low intensity [13]. A small number of patients exhibits diffusely increased activity in one or both breasts, which may be related to an increase of hormone levels at the time of imaging. With the equipment of breast-specific gamma imaging or molecular breast imaging, ${ }^{99 \mathrm{~m}} \mathrm{Tc}$ sestamibi has been reported to have a sensitivity greater than $80 \%$ [14]. A negative scan in the presence of a palpable lesion is also significant, making breast cancer possible but unlikely. Moreover, the addition of ${ }^{99 \mathrm{~m}} \mathrm{Tc}$ sestamibi gamma imaging to mammography significantly increased the detection of nodenegative breast cancer in dense breasts [15]. Of note, Collarino and colleagues recently reported that ${ }^{99} \mathrm{Tc}$-sestamibi uptake during neoadjuvant chemotherapy seems highly sensitive for the prediction of nonresponsiveness [16]. In a study, authors revealed that the sensitivity of mammography alone was $27 \%$, while the sensitivity of combined mammography and dedicated sestamibi imaging was $91 \%$ in at-risk women $[17,18]$. In spite of these promising results, the use of ${ }^{99 \mathrm{~m}} \mathrm{Tc}$ sestamibi high-resolution SPECT as a screening procedure is not currently recommended by international guidelines. However, the interpretation of results obtained by ${ }^{99 \mathrm{~m}} \mathrm{Tc}$ sestamibi high-resolution SPECT, in the light of the new discoveries concerning both the presence and the role of BOLCs in breast cancer, could provide innovative clinical indication for the large-scale use of this technology. Indeed, actually no clinicalinstrumental investigation allows to classify breast cancers based on their capacity to form bone metastatic lesions. In a recent study, authors correlated the presence of BOLCs with the mammographic characteristics of breast lesions as microcalcifications [10]. Although encouraging, data reported by Bonfiglio et al. were influenced by the numerous variables of the mammographic examination, especially with regard to the analysis of microcalcifications [10]. In fact, only the crush stone calcifications seemed to be related to the presence of BOLCs. On the other hand, the results of $\left({ }^{99} \mathrm{Tc}\right)$ sestamibi high-resolution SPECT analysis could highlight the real-time activity of BOLCs, rather than the presence of calcium crystals produced by them, thus producing more comparable data with the histological and molecular ones, obtained from biopsies. For this reason, large-cohort studies will be necessary in order to support the introduction of ${ }^{99 \mathrm{~m}} \mathrm{Tc}$ sestamibi analysis as a screening of breast cancer lesions. In particular, it will be essential to identify significant correlations among morphological, ultrastructural, immunohistochemical, molecular features of BOLCs and ${ }^{99 \mathrm{~m}} \mathrm{Tc}$ sestamibi in vivo uptake.

In conclusion, if verified, prospectives here reported may have important consequences for the management of breast cancer. The early identification of lesions with high metastatic potential by ${ }^{99 \mathrm{~m}} \mathrm{Tc}$ sestamibi high-resolution SPECT analysis could be considered a new frontier for diagnosis and/or therapy of breast lesions. In fact, BOLCs might also represent a target for the development of innovative therapies directed against BOLC antigens with the aim to block/prevent the breast cancer progression or bone lesions formation. 


\section{Conflict of interest}

There are no potential conflicts of interest relating to the manuscript (for each authors), and there were no extramural sources supporting this research (excluding sources already declared). The study is original and the manuscript has not been published yet and is not being considered for publication elsewhere in any language either integrally or partially, except as an abstract. All authors have agreed with the submission in its present (and subsequent) forms.

\section{Financial \& competing interests disclosure}

The authors have no relevant affiliations or financial involvement with any organization or entity with a financial interest in or financial conflict with the subject matter or materials discussed in the manuscript. This includes employment, consultancies, honoraria, stock ownership or options, expert testimony, grants or patents received or pending, or royalties.

No writing assistance was utilized in the production of this manuscript.

\section{References}

1. Cancer Genome Atlas Network. Comprehensive molecular portraits of human breast tumours. Nature 490(7418), 61-70 (2012).

2. Donovan D. Metastatic breast cancer epidemiology and management with a focus on taxanes. Clin. J. Oncol. Nurs. 17(Suppl. 5-8) (2013).

3. Hess G, Barlev A, Chung K, Hill JW, Fonseca E. Cost of palliative radiation to the bone for patients with bone metastases secondary to breast or prostate cancer. Radiat. Oncol. 7, 168 (2012).

4. Scimeca M, Antonacci C, Toschi N et al. Breast osteoblast-like cells: a reliable early marker for bone metastases from breast cancer. Clin. Breast Cancer 18(4), e659-e669 (2018).

5. Scimeca M, Bonfiglio R, Montanaro M, Bonanno E. Osteoblast-like cells in human cancers: new cell type and reliable markers for bone metastasis. Future Oncol. 14(1), 9-11 (2018).

6. Scimeca M, Antonacci C, Colombo D, Bonfiglio R, Buonomo OC, Bonanno E. Emerging prognostic markers related to mesenchymal characteristics of poorly differentiated breast cancers. Tumour Biol. 37(4), 5427-5435 (2016).

7. Yang W, Guo D, Harris MA et al. Bmp2 in osteoblasts of periosteum and trabecular bone links bone formation to vascularization and mesenchymal stem cells. J. Cell Sci. 126(Pt 18), 4085-4098 (2013).

8. Scimeca M, Salustri A, Bonanno E et al. Impairment of PTX3 expression in osteoblasts: a key element for osteoporosis. Cell Death Dis. 8(10), e3125 (2017).

9. Scimeca M, Giannini E, Antonacci C, Pistolese CA, Spagnoli LG, Bonanno E. Microcalcifications in breast cancer: an active phenomenon mediated by epithelial cells with mesenchymal characteristics. BMC Cancer 14, 286 (2014).

10. Bonfiglio R, Scimeca M, Toschi N et al. Radiological, histological and chemical analysis of breast microcalcifications: diagnostic value and biological significance. J. Mammary Gland Biol. Neoplasia 23, 89 (2018).

11. Scopinaro F, Schillaci O, Scarpini M et al. Technetium-99 m sestamibi: an indicator of breast cancer invasiveness. Eur. J. Nucl. Med. 21(9), 984-987 (1994).

12. Komarova SV, Ataullakhanov FI, Globus RK. Bioenergetics and mitochondrial transmembrane potential during differentiation of cultured osteoblasts. Am. J. Physiol. Cell Physiol. 279(4), C1220-C1229 (2000).

13. Schillaci O, Spanu A, Danieli R, Madeddu G. Molecular breast imaging with gamma emitters. Q. J. Nucl. Med. Mol. Imaging 57(4), 340-351 (2013).

14. Colella AC, Scopinaro F, Schillaci O et al. 99mTc-sestaMIBI breast scintigraphy. Tumori 83(2), 520-522 (1997).

15. Schillaci O, Danieli R, Romano P, Santoni R, Simonetti G. Scintimammography for the detection of breast cancer. Expert Rev. Med. Devices 2(2), 191-196 (2005).

16. Collarino A, de Koster EJ, Valdés Olmos RA, de Geus-Oei LF, Pereira Arias-Bouda LM. Is technetium-99 m sestamibi imaging able to predict pathologic nonresponse to neoadjuvant chemotherapy in breast cancer? A meta-analysis evaluating current use and shortcomings. Clin. Breast Cancer 18(1), 9-18 (2018).

17. Mettler FA Jr. Non-PET neoplasm imaging and radioimmunotherapy. In: Essentials of Nuclear Medicine Imaging (Sixth Edition). 345-360 (2012).

18. Collarino A, Valdés Olmos RA, van Berkel LGAJ et al. The clinical impact of molecular breast imaging in women with proven invasive breast cancer scheduled for breast-conserving surgery. Breast Cancer Res. Treat. 169(3), 513-522 (2018). 
